\title{
Anti-fatigue Properties of Icariin Isolated from Epimedium Brevicornum Maxim in Mice
}

\author{
Changjun $\mathrm{Li}^{1, \text { a }}$ and Ang $\mathrm{Li}^{2, \mathrm{~b}}$ \\ ${ }^{1}$ Huangshan University, Huangshan, 245041, China \\ ${ }^{2}$ Hefei University of Technology Xuancheng Campus, Xuancheng, 242000, China \\ alichangjunm@yeah.net, ${ }^{b} 349738728 @ q q . c o m$
}

\begin{abstract}
Keywords: icariin; anti-fatigue; forced swimming test; exhaustive swimming times; mice.
Abstract. Aim of the present study was to evaluate the anti-fatigue properties of icariin in mice. The animals were divided into four groups, namely the control, low-dose icariin treated, medium-dose icariin treated, and high-dose icariin treated groups, which received distilled water and 5, 10 and 20 $\mathrm{mg} / \mathrm{kg}$ icariin, respectively, by oral gavage. After 28 days, the forced swimming test was performed, along with the determination of blood lactic acid (BLA), serum urea nitrogen (SUN), superoxide dismutase (SOD), glutathione peroxidase (GPx) and malondialdehyde (MDA). The results showed that icariin could extend the exhaustive swimming times, as well as decrease the BLA, SUN and serum MDA levels, while increasing the serum SOD and GPx levels. Thus, the results demonstrated that icariin has anti-fatigue properties.
\end{abstract}

\section{Introduction}

Fatigue may be defined as a situation in which the capacity for work is diminished and efficiency of accomplishment reduced [1]. Fatigue is divided into physical and mental fatigue. Physical fatigue is the inability to continue functioning at a level commensurate with normal ability, while mental fatigue may manifest in the form of decreased attention, reduced ability to concentrate, or somnolence [2]. There are several theories about the causes of fatigue, such as exhaustion theory, homeostasis disturbance theory, clogging theory, free radical theory and catastrophe theory [3]. Among these, free radical theory has attracted much attention in anti-fatigue study. Free radical and reactive oxygen species (ROS) production during exercise contributes to fatigue, and antioxidant treatment might be a therapeutic approach [4].

Epimedium brevicornum Maxim (E. brevicornum) from many species of the genus Epimedium (Berberidaceae), a commonly used Chinese medicine, has been widely used in China in the treatment of cardiovascular diseases, infertility, impotence, amnesia, lumbago, arthritis, numbness and weakness of the limbs for thousands of years [5,6]. The main bioactive components in $E$. brevicornum are epimedin epimedin A, epimedin B, epimedin C and icariin [7]. Recently, icariin $\left(\mathrm{C}_{33} \mathrm{H}_{40} \mathrm{O}_{15}\right.$ : molecular weight: 676.67) has been reported to have a variety of pharmacological actions, including antioxidant, anti-inflammatory, anti-depressant, anti-obesity, estrogen-like and immunoregulatory activities [8]. However, the effects of icariin on physical fatigue have not been investigated thus far. Therefore, the current study was designed to evaluate the anti-fatigue properties of icariin using forced swimming test in mice.

\section{Materials and Methods}

Chemicals and reagents. Icariin were purchased from Chengdu Herb Purify Co. Ltd. (Chengdu, China). The assay kits for blood lactic acid (BLA), serum urea nitrogen (SUN), superoxide dismutase (SOD), glutathione peroxidase (GPx) and malondialdehyde (MDA) were purchased from Jiancheng Institute of Biotechnology (Nanjing, China). All other chemicals and reagents, used in the present study, were of analytical reagent grade with high purity and were purchased from Anhui Chemical Reagent Company Limited (Hefei, China). The double distilled water were used to prepare all solutions. 
Selection of animal and care. Male Kunming mice (body weight $20 \pm 2$ g) were obtained from Experimental Animal Center of Anhui Province (Hefei, China). The animals were acclimatized to the environment ( $22 \pm 2{ }^{\circ} \mathrm{C}, 55 \pm 10 \%$ relative humidity, and $12 \mathrm{~h}$ dark/light cycle) for 1 week prior to experimental use. The animals were maintained with standard pellet diet, while tap water was available ad libitum. All the experimental procedures were performed according to the China legislation the use and care of laboratory animals, and were approved by the Ethical Committee of Huangshan University (Huangshan, China).

The animals were divided into four groups and each group consisted of eight mice. (1) Control (C) group: mice were allowed to free access to standard pellet diet and treated with distilled water (2.0 $\mathrm{ml}$ ). (2) Low-dose icariin treated (LI) group: mice were allowed to free access to standard pellet diet and treated with icariin solution (5 mg/kg). (3) Medium-dose icariin treated (MI) group: mice were allowed to free access to standard pellet diet and treated with icariin solution $(10 \mathrm{mg} / \mathrm{kg})$. (4) High-dose icariin treated (HI) group: mice were allowed to free access to standard pellet diet and treated with icariin solution $(20 \mathrm{mg} / \mathrm{kg}$ ).Treatments were administered by oral gavageonce daily for 28 days. Icariin solution were prepared through dissolving it in $2.0 \mathrm{ml}$ distilled water.

Forced swimming test. After the final icariin or distilled water treatment, the mice were allowed to rest for $30 \mathrm{~min}$, and then they were subjected to the forced swimming test by our previously described method [9]. Briefly, the mice were placed individually into acrylic plastic pool (50 cm in length, 38 $\mathrm{cm}$ in width and $38 \mathrm{~cm}$ in high) filled with water to a depth of $30 \mathrm{~cm}$. The temperature of the water was maintained at $25 \pm 1^{\circ} \mathrm{C}$. Each mouse was weighted using a lead wire bundle attached to the tail at $5 \%$ of the body weight. The exhaustion criteria were accepted when the mice uncoordinatedly moved and stayed under the water for 10 seconds without swimming at the surface.

Blood biochemical analyses. After the forced swimming test, the mice were immediately anesthetised using ethyl ether and sacrificed by exsanguination via the abdominal aorta. Blood were collected and serum was separated by centrifugation $(1200 \times \mathrm{g}$ for $10 \mathrm{~min})$ at $4{ }^{\circ} \mathrm{C}$. Samples were stored at $-80{ }^{\circ} \mathrm{C}$ to determine BLA, SUN, SOD, GPx and MDA levels. All the blood biochemical parameters were measured according to the recommended procedures provided by the assay kits.

Statistical analyses. SPSS 15.0 software was used for statistical analyses. Values were reported as means \pm SD. Statistical comparisons of the differences were performed using one way analysis of variance for repeated measures combined with the Newman-Keuls post hoc test. Group means were considered to be significantly different at $\mathrm{p}<0.05$.

\section{Results and Discussion}

Effects of icariin on the exhaustive swimming times of mice. Forced swimming test is one of the most valid models for evaluating anti-fatigue properties in animals [10]. As shown in Fig. 1, the exhaustive swimming times of LI, MI and HI groups were significantly higher than that of the C group $(p<0.05)$. This data clearly reveal that icariin could elevate exercise tolerance and relieve fatigue.

Effects of icariin on BLA and SUN of mice. Fatigue after exercise can be evaluated by some important blood biochemical parameters, including lactic acid and serum urea nitrogen. Lactic acid is the glycolysis product of carbohydrate under an anaerobic condition, and glycolysis offers main energy for intense exercise in a short time [11]. Many organs, especially the liver and skeletal muscle, help remove lactic acid from the blood. However, intense exercise can increase BLA production to a point that exceeds the rate of lactate removal, this condition resulting in fatigue [12]. SUN is another blood biochemical index related to fatigue, which is formed in the liver as the end product of protein metabolism. When the body is unable to obtain sufficient energy from sugar and fat catabolism, it utilizes proteins and amino acids that have a stronger catabolism [10]. As shown in Fig. 2, the BLA levels of LI, MI and HI groups, as well as the SUN levels of MI and HI groups were significantly lower than that of the $C$ group $(p<0.05)$. This data clearly reveal that icariin could prevent the accumulation of BLA and might reduce protein catabolism for energy, and thus retard the occurrence of fatigue. 


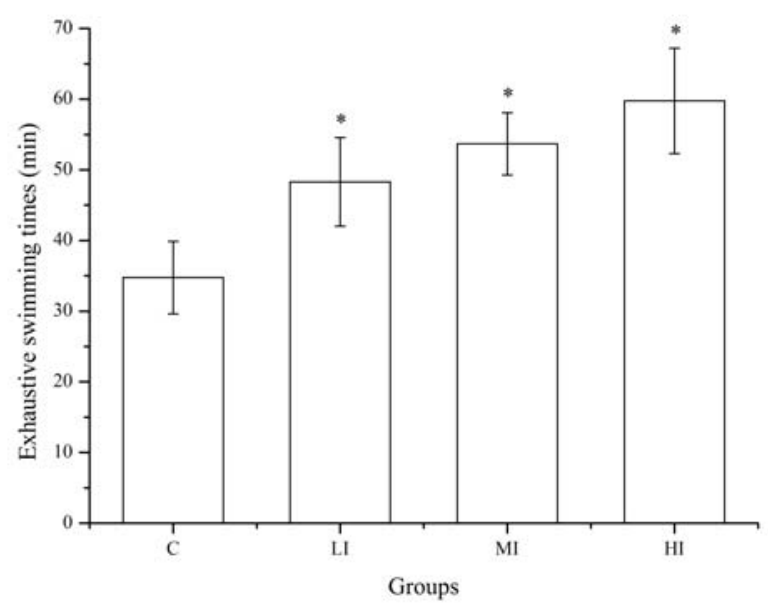

Fig. 1. Effects of icariin on the exhaustive swimming times of mice. Note, Values are expressed as means $\pm \mathrm{SD} ;{ }^{*} \mathrm{p}<0.05$, compared with $\mathrm{C}$ (control) group

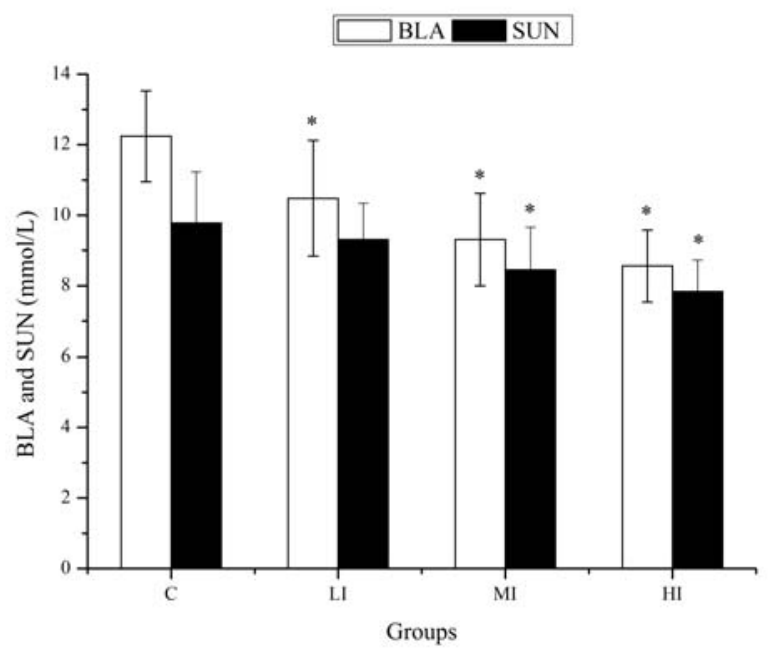

Fig. 2. Effects of icariin on BLA and SUN of mice. Note, Values are expressed as means \pm SD; ${ }^{*}<0.05$, compared with $\mathrm{C}$ (control) group

Effects of icariin on the serum SOD, GPx and MDA of mice. Exhaustive exercise is often associated with an increase in the production of free radical and ROS in various tissues, which may overwhelm the capacity of the antioxidant defense systems, and oxidative stress occurs [13]. Accumulating evidence indicates that the accumulation of free radical and ROS are responsible for exercise-induced protein oxidation and contribute strongly to physical fatigue [14]. The primary antioxidant enzymes include SOD and GPx. These antioxidant enzymes become weaker during fatigue and other disease conditions. In addition, exhaustive exercise-induced free radical and ROS attack the membrane lipids and results in lipid peroxidation product formation [15]. MDA is frequently used as an indicator of lipid peroxidation. Therefore, the improvement in the activities of these antioxidant enzymes, along with decrease in the MDA levels, could help to fight against fatigue. As shown in Fig. 3, the serum SOD and GPx levels of LI, MI and HI groups were significantly higher than that of the $\mathrm{C}$ group $(\mathrm{P}<0.05)$. Icariin also affected serum MDA levels of LI, MI and HI groups significantly lower than that of the $C$ group $(P<0.05)$. This data clearly reveal that icariin could enhance antioxidant enzymes activities and reduce lipid per-oxidation, which suggested that the anti-fatigue properties of icariin probably occurs through protection of corpuscular membranes by preventing lipid oxidation via modifying activities of several antioxidant enzymes [16]. Additional studies are needed to confirm this hypothesis. 


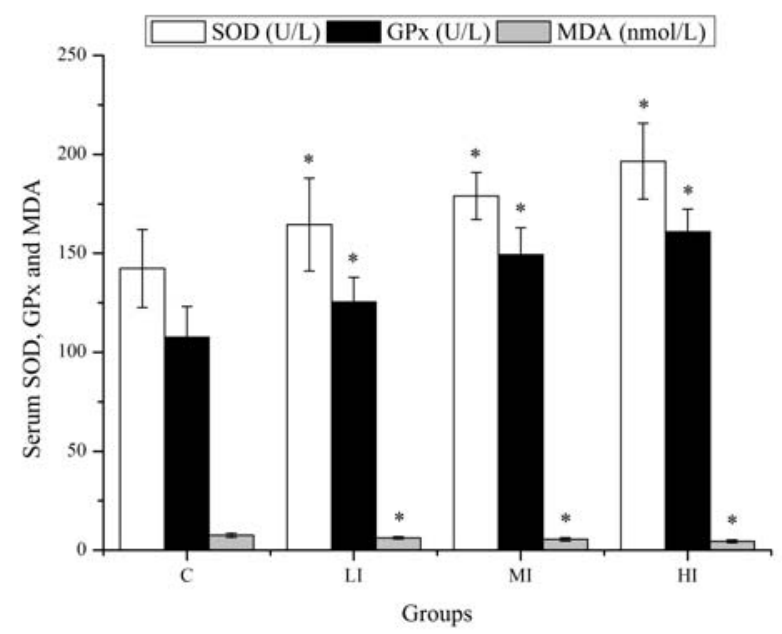

Fig. 3. Effects of icariin on the serum SOD, GPx and MDA of mice. Note, Values are expressed as means $\pm \mathrm{SD} ;{ }^{*} \mathrm{p}<0.05$, compared with $\mathrm{C}$ (control) group

\section{Conclusion}

This study demonstrated that icariin has anti-fatigue properties by increasing exhaustive swimming times, as well as decreasing BLA and SUN levels. Furthermore, It also improved the serum SOD and GPx levels and decreased the serum MDA levels in the mice, which may be one of the pathways behind the icariin's anti-fatigue properties.

\section{References}

[1] G. Zhang, S.M. Zhou, Q.Y. Huang and Y.Q. Gao: Trop. J. Pharm. Res Vol. 11 (2012), p. 209.

[2] A. Panossian and G. Wikman: Curr. Clin. Pharmacol Vol. 4 (2009), p. 198

[3] X. Wang, R. Xing, Z. Chen, H. Yu, R. Li and P. Li: Food Funct Vol. 5(2014), p. 2113

[4] Y. Lin, H.L. Liu, J. Fang, Y.K. Xiong and K. Yuan: Food Chem. Toxicol Vol. 68 (2014), p. 290

[5] D.W. Zhang, Y. Cheng, M.S. Yang and X.S. Yao: Phytomedicine Vol. 15 (2008), p. 55

[6] H.B. Li and F. Chen: J. Chromatogr. Sci Vol. 47 (2009), p. 337

[7] C.W. Chen, Q.P. Dai, Y.Q. Chen and T. Che: Mediat. Inflamm Vol. 2016 (2016), p. 9529630

[8] H.F. Zhang, T.S. Yang, Z.Z. Li and Y. Wang: Ultrason. Sonochem Vol. 15 (2008), p. 376

[9] C.J. Li, X.L. Wu, X.J. Lou, Y.J. Wu and H.Y. Wang: J. Anim. Vet. Adv Vol. 11 (2012), p. 1627

[10] B. Qi, L. Liu, H. Zhang, G.X. Zhou, S. Wang, X.Z. Duan, X.Y. Bai, S.M. Wang and D.Q. Zhao: J. Ethnopharmacol Vol. 28 (2014), p. 430

[12] C.J. Li and A. Li: Appl. Mecha. Mater Vol. 730 (2015), p. 241

[12] S. Lee, Y. You, H.G. Yoon, K. Kim, J. Park, S. Kim, J.N. Ho, J. Lee, S. Shim and W. Jun: Biosci. Biotechnol. Biochem Vol. 75 (2011), p. 349

[13] E. Jówko, J. Sacharuk, M. Charma and R. Charmas: Nutr. Res Vol. 31 (2011), p. 813

[14] K.Y. Su, C.Y. Yu, Y.W. Chen, H.F. Wu and Y.L. Chen: Int. J. Med. Sci Vol. 29 (2014), p. 528

[15] F. Yan and H. Hao: J Biol Res (Thessalon) Vol. 23 (2016), p. 7

[16] B. Lamou, G.S. Taiwe, and P.V. Tan: Oxid. Med. Cell Longev Vol. 2016 (2016), p. 3517824 